CASE REPORT

\title{
Vertebral artery dissection and cerebellar infarction following chiropractic manipulation
}

\author{
W-L Chen, C-H Chern, Y-L Wu, C-H Lee
}

Emerg Med J 2006;23:e1 (http://www.emjonline.com/cgi/content/full/23/1/e1). doi: 10.1136/emj.2004.015636

\begin{abstract}
Vertebral artery dissection (VAD) associated with chiropractic cervical manipulation is a rare but potentially disabling condition. In this report, we present a young patient manifesting with repeated vertigo. Owing to the initial misdiagnosis, the patient later developed cerebellar stroke with inability to stand or walk. Vertigo and disequilibrium are the usual presenting symptoms of this condition, which can result from inner ear or vestibular nerve dysfunction, vertebrobasilar insufficiency, and even lethal cerebellar infarction or haemorrhage; these last two, although rarely seen in young adults, can be caused by traumatic or spontaneous arterial injury, including injury secondary to chiropractic cervical manipulation. A number of cases of VAD associated with chiropractic cervical manipulation have been reported, but rarely in the emergency medicine literature. We present a case of this rare occurrence, and discuss the diagnostic piffalls.
\end{abstract}

$\mathrm{V}$ ertigo and dizziness are among the commonly encountered symptoms presented by patients attending the emergency department (ED). Some of these patients, especially the elderly, have vertebrobasillar insufficiency (VBI) and even lethal cerebellar or brainstem infarct or haemorrhage. However, complications arising from chiropractic manipulation, especially vertebral artery dissection (VAD), may be seen in younger patients, causing VBI or cerebellar or brainstem infarct. This condition is rare but clinically significant, as its rarity and sometimes clinically atypical presentation can lead to misdiagnosis, with possible lethal outcome.

\section{CASE REPORT}

A 28 year old man in good health visited a chiropractor for treatment of neck pain for about 2 weeks. Following one of these chiropractic manipulation sessions, he began experiencing vertigo, and attended our ED immediately. On arrival, the symptoms had disappeared spontaneously. Physical and neurological examinations were essentially normal, and he was discharged with an appointment made for follow up at the outpatient clinic. The following day, he experienced another episode of vertigo, along with loss of balance, blurred vision, and mild numbness and weakness of the left extremities. He was again referred to our ED. Physical examination revealed a young man who appeared well, and was alert and cooperative except for an inability to tolerate sudden changes in position. Blood pressure was $146 / 76 \mathrm{mmHg}$, pulse rate was 90 beats/min, respiratory rate 18 breaths/min and ear temperature was $37.0^{\circ} \mathrm{C}$. He denied any episodes of headache, syncope, head or neck trauma, and use of recreational drugs. Neurological examinations, including motor and sensory function, were essentially nonfocal; however, the patient could not stand or walk because of vertigo. Cerebellar tests including finger to nose, rapid alternating movement, and heel to shin were impaired on the right side. Routine laboratory studies including blood tests and electrolytes were within normal limits. Electrocardiography showed normal sinus rhythm, and cervical spine radiographs were normal. While retaking his history, we found that he had experienced several episodes of vertigo after cervical manipulation, thus, under high suspicion of cerebellar stroke associated with acute vascular injury, Doppler ultrasound of the extracranial artery was performed, which showed trickle flow over right distal vertebral artery. Magnetic resonance imaging of the brain revealed a tiny lesion over the right cerebellum with high intensity signal on T2 weighted image (fig lA), which showed as a bright area on diffusion weighted images and dark on apparent diffusion coefficient mapping, most likely a tiny recent or acute infarction. Angiography of the vertebral artery revealed occlusion of the right distal vertebral artery near the craniocervical junction (fig $1 \mathrm{~B}$ ).

Vertebral artery stenting was tried, but failed to pass the distal true lumen. The patient was admitted to the neurological intensive care unit and placed on anticoagulant therapy (heparin). On the eighth day, he was discharged without any sequelae (NIHSS: 0 ) and followed up regularly at our outpatient clinic with oral anticoagulant therapy.

\section{DISCUSSION}

Chiropractic spinal manipulation is a popular alternative therapy for neck and back pain, which is performed by chiropractors, who tend to believe that it is not associated with relevant risks. However, in 1947, Pratt-Thomas and Berger reported two patients who became unconscious during chiropractic adjustment and died in under 24 hours. ${ }^{1}$ Since then, the risk factors and complications of chiropractic manipulation have been under discussion, and the opinion that chiropractic spinal manipulation is risk free has been contradicted by findings in the published literature. ${ }^{2-7}$ The most frequently reported complication is posterior circulation stroke, usually related to $\mathrm{VAD}$, occurring during or shortly after cervical manipulation. ${ }^{236}$

Some diagnostic pitfalls should be noted for this condition. Firstly, because of the unfamiliarity and rarity of the condition, there is a possibility that medical staff will fail to elicit a history of cervical manipulation. The specific questions regarding spinal manipulation were not included when the patient made his first visit to the ED, and it was not until the second visit, with the patient showing recurrent vertigo and disequilibrium, which might be the signs of VBI, that the cervical manipulation history was discovered. Secondly, this condition may show a fluctuating course. The aetiology of vertigo is classically separated into peripheral and central types. Because the neurological examination of our patient

Abbreviations: $E D$, emergency department; $V A D$, vertebral artery dissection; VBI, vertebrobasillar 

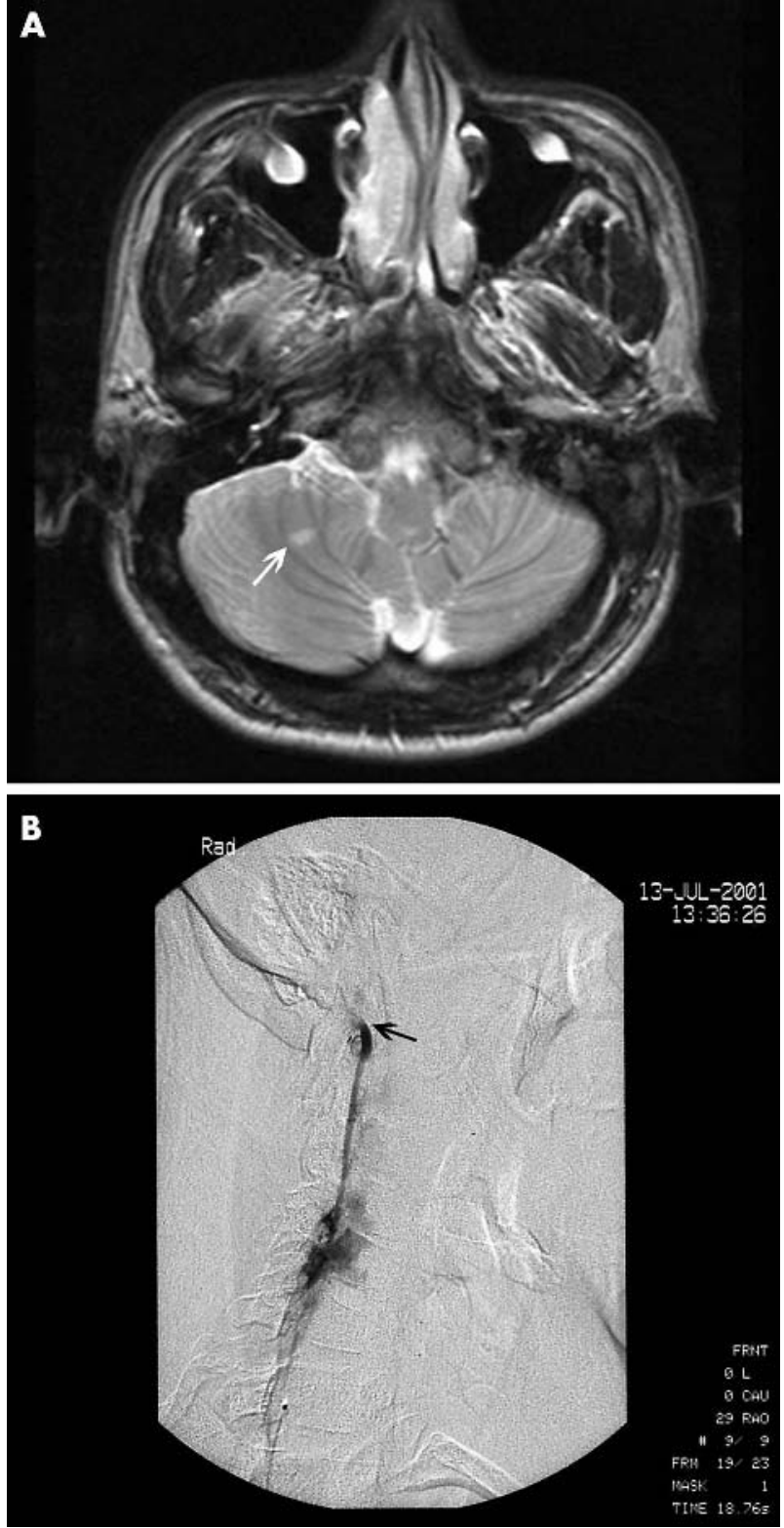

Figure 1 (A) Magnetic resonance image (T2 weighted) showing a tiny lesion over the right cerebellum (arrow); (B) angiography showing occlusion of the right distal vertebral artery near the craniocervica junction (arrow).

on the first visit was essentially normal, and he was a relatively young patient without any risk factors of stroke, he was discharged under the impression of peripheral vertigo. A fluctuating clinical course, as in our case, with normal findings in at certain time points in the course of the condition, may also contribute to misdiagnosis.

Some authors are of the opinion that brain ischaemia is delayed by hours or even weeks after injuries, thus the presence of such a delay provides a time window during which measures could be undertaken to prevent thromboembolism and to maintain vessel patency. ${ }^{8}$ The vertebrobasilar infarction associated with VAD may significantly contribute to disability in surviving patients, and may even have legal implications. Early diagnosis and management is therefore essential. Anticoagulation with heparin followed by warfarin is the treatment of choice. Surgical intervention is indicated only for those patients with persistent symptoms refractory to medical therapy. Endovascular treatment (balloon angioplasty followed by placement of stents) has largely supplanted surgery as the initial therapy of choice in cases where medical therapy fails or is contraindicated, and even instead of medical therapy for many asymptomatic patients. ${ }^{9}$

Smith et al have suggested that chiropractic manipulation independently increases the risk of VAD with stroke by approximately six fold. ${ }^{10}$ Rates of VBD following cervical manipulation from 1 in 10000 to 1 in 2 million have been reported, ${ }^{611}{ }^{12}$ but the exact incidence is still unknown. However, this diagnosis should be considered in patients presenting with recent onset vertigo and loss of balance, and history taking should include questions on presence or absence of recent spinal manipulation.

\section{Authors' affiliations}

W-L Chen, Department of Emergency Medicine, Cathay General Hospital, and Institute of Emergency and Critical Care Medicine, National Yang-Ming University, Taipei, Taiwan, Republic of China C-H Chern, Department of Emergency Medicine, Veterans General Hospital, Taipei, Taiwan, Republic of China

Y-L Wu, Department of Emergency Medicine, Cathay General Hospital, Taipei, Taiwan, Republic of China

C-H Lee, Institute of Emergency and Critical Care Medicine, National Yang-Ming University, Taipei, Taiwan, Republic of China

Competing interests: none declared

Correspondence to: Dr W-L Chen, Department of Emergency Medicine, Cathay General Hospital, 280 Jen-Ai Road, Section 4, Taipei 106, Taiwan, Republic of China; weilung.chen@msa.hinet.net

Received 27 February 2004

Revised 27 March 2004

Accepted for publication 1 April 2004

\section{REFERENCES}

1 Pratt-Thomas HR, Berger KE. Cerebellar and spinal injuries after chiropractic manipulation. JAMA 1947;133:600-3.

2 Setvinson C, Honan W, Cooke B, et al. Neurological complications of cervical spine manipulation. J R Soc Med 2001;94:107-10.

3 Lee KP, Carlini WG, McCormick GF, et al. Neurologic complications following chiropractic manipulation: A survey of California neurologists. Neurology 1995;45:1213-15.

4 Siegel D, Neiders T. Vertebral artery dissection and pontine infarct after chiropractic manipulation. Am J Emerg Med 2001;19:171-2.

5 Devereaux MW. The neuro-ophthalmologic complications of cervical manipulation. J Neuro-Ophthalmol 2000;20:236-9.

6 Assendelft WJJ, Bouter LM, Knipschild PG. Complications of spinal manipulations: a comprehensive review of the literature. J Fam Pract 1996:42:475-80

7 Senstad O, Leboeuf-Yde, Borchgrevink C. Frequency and characteristics of side effects of spinal manipulative therapy. Spine 1997;22:435-41.

8 Tulyapronchote R, Selhorst JB, Malkoff MD, et al. Delayed sequelae of vertebral artery dissection and occult cervical fractures. Neurology 1994;44:1397-9

9 Schievink WI. The treatment of spontaneous carotid and vertebral artery dissections. Curr Opin Cardiol 2000;15:316-21.

10 Smith WS, Johnston SC, Skalabrin EJ, et al. Spinal manipulation therapy is an independent risk factor for vertebral artery dissection. Neurology 2003;60:1424-8

11 Rothwell DM, Bondy SJ, Williams J. Chiropractic manipulation and stroke. A population-based case-control study. Stroke 2001;32:1054-60.

12 Meeker WC, Haldeman S. Chiropractic: a profession at the crossroads of mainstream and alternative medicine. Ann Intern Med 2002;136:216-27. 